\title{
Die Wanderung der Alkaloide aus dem Pfropfreise in die Unterlage.
}

Von Erast Schmidt and Arthur Meyer.

(Eingegangen den 18. VI. 1907.)

Die Fragen, ob die Alkaloide in der Pflanze wandern and welche Wege sie bei dieser Wanderung einschlagen, sind noch nicht gelöst. Sie wurden bei den unter unserer Leitung von Feldhaus (1903) and von Kircher (1905) ausgefuhrten Arbeiton in folgender Weise berührt:

Es laßt sich schon mikrochemisch erkennen, daß in den Blattern von Datura Stramonium, ebenso in denen von Hyoscyamus (Siim Jensen, 1901), die Alkaloide im Parenchym der Leitbündel viel reichlicher vorkommen als im Assimilationsparenchym. So fand auch Feldhaus in den Mittelnerven and Sekundurnerven von Datura $1,39 \%$, im Mesophyll mit den kleineren Nerven nur $0,48 \%$ Alkaloid, bezogen auf Trockensubstanz, im Blattgtiele etwas weniger Alkaloid als im Mittelnerven. Im allgemeinen verhielt sich der Alkaloidgehalt von Blattspreite mit Nerven höherer Ordnang : Mittel- + Sekundarnerven : Blattstiel $=1: 3: 1,5$.

Feldhaus (19. B. S. 82) schnitt nun von einer groberen Anzahl von Laubblättern die Spreitenbälften rechts and links vom Mittelnerven $a b$ und lie $B$ die Blattstiele mit den daran sitzenden Mittelnerven der Blätter vom 30. Juli bis 28. Augast an den Pfianzen. Danach fand er in Mittelrippe nad Blattstiel zasammen nur $029 \%$ Alkaloid, also viel weniger als in der normalen Blattspreite.

Kircher verfolgte diese Erscheinung weiter, indem or folgendermaßen verfuhr: Zuerst sammelte or von zwel verschiedenen Beeten (I and III) von Datura Stramonium je nngetähr 300 ganze Blatter. Zweitens schnitt er von ungefahr 700 Blattern des Beetes I die Spreiten rechts und links rom Mittelnerven vollig ab und sammelte sogleich 300 Blattstiele + Mittelnerven; die ubrigen Blattatiele + Mittelnerven ließ er an den Pflanzen sitzen und sammelte sie erst nach fünf und nach acht Tagen, nach welcher Zeit manche Blattstiele abgefallen, manche erkrankt waren. Drittens schnitt er von einer gleichen Anzahl von Blättern des anderen Beetes (No. III) die Spreitenteile bis auf einen Streifen von 2-3 mm, welchen er an jeder Seite des Mittelnerven stehen lie $\emptyset$, ab und verfuhr damit wie vorher gesagt; es hielten sich diese Blattstiele + Mittelnerven gut nod fielen nicht ab. Als er die Trockensubstanz aller Proben untersuchte, fand er folgendes: 


\begin{tabular}{|c|c|c|c|}
\hline \\
\hline Gadze biatter & III $=0,85 n$ & $n$ & \\
\hline $\begin{array}{c}\text { Direkt gesammelte } \\
\text { Stiele }+ \text { Mittelnerven }\end{array}$ & $\begin{aligned} \mathrm{I} & =0,8 \\
\mathrm{III} & =0,81\end{aligned}$ & " & $\begin{array}{l}\text { Spreite völlig entfernt. } \\
2-3 \text { mm Spreite am } \\
\text { Mittelnerven. }\end{array}$ \\
\hline $\begin{array}{l}\text { Nach fünf Tagen } \\
\text { gesammelt }\end{array}$ & $\left\{\begin{aligned} \mathrm{I} & =0,65, \\
\mathrm{III} & =0,79 n\end{aligned}\right.$ & n & \\
\hline $\begin{array}{l}\text { Nach acht Tagen } \\
\text { gesammelt }\end{array}$ & $\left\{\begin{aligned} I & =0,5 n \\
I I I & =0,78 n\end{aligned}\right.$ & n & \\
\hline
\end{tabular}

Es ist damit bewiesen, daß der Alkaloidgehalt an der Pflanze sitzender Blattstiele + Mittelnerven, denen die Spreiten genommen warden, mit der Zeit mehr und mehr abnimmt, daß aber schon ein geringer Teil der ansitzenden Spreite diese Abnahme stark herabsetzt. Wenn dieses Resultat auch nicht beweist, daß das Hyoscyamin ans dem Stiele aus- und in die Achse einwandert, so liegt doch die Annahme nahe, daß die Abnahmo des Alkaloides im Stiele anf einer Answanderung des Alkaloides beruht.

Demgegentuber schien die Frage, ob die Alkaloide von dem Orte ihrer Entstehung wegwandern kőnnen, durch einen von Strasbarger (1885 und 1906) angestellten Versuch gelöst za sein. Durch Strasburger veranlaßt, untersuchte Klinger $800 \mathrm{~g}$ Kartoffelknollen, welche an einer dorch ein Pfropfreis von Datura Stramonium ernährten Unterlage von Solanum tuberosum entstanden waren, und fand darin Atropin. Strasburger (1885, S. XXXIX) sagt: „Er (Klinger) fand Atropin, wenn auch nur in außerst geringen Mengen; nach seiner Schătzang würden die $800 \mathrm{~g}$ Knollen kaum einige Milligramm Atropin enthalten haben." Klinger unterwarf ubrigens auch $600 \mathrm{~g}$ gew licher Kartoffelknollen der Untersuchung und fand darin weder Atropin noch ein dem Atropin ähnliches Alkaloid.

Es schien uns nun für die Frage der Alkaloidwanderung zuerst eine Kontrolle der vorliegenden Angaben von Interesse zn sein. Da eine Ptropfung von Datura Stramonium im Frúhjahr 1906 gut angewacbsen war, beschlossen wir die zu erwartenden Kartoffeln dazu za benutzen und im kommenden Frühjahr die am Schlusse dieser Notiz anfgeführten Fragen zu beantworten. Währeud der Zeit sind nun weiter zwei hierher gehörende Arbeiten erschienen, zuerst die von Grafe und Linsbauer (1906).

Grafe and Linsbauer experimentierten mit Nicotiana affinis und Nicotiana Tabacum, die sie wechselweise anfeinander pfropften. Sie betrachten $N$. affinis als nikotinfrei oder als so nikotinarm, daß sie ihren Nikotingehalt nicht in Betracht ziehen; da aber $N$. affinis 
Nikotin enthălt und anzanehmen ist, daß ihr Nikotingehalt ahnlichen Schwankungen anterliegt wie der von $N$. Tabacum, deren Alkaloidgehalt zwischen $0,7 \%$ und $5 \%$ schwankt, so ist dieses Vorgehen wohl etwas nnkritisch and laßt leider Zweifel an der Znverlässigkeit der Resultate entstehen. Es häte zuvor eine grobere Anzahl von Individuen der benutzten $N$. affinis genau anf ihren Alkaloidgehalt untersucht werden missen.

Die Versuche der Autoren zeigten nun, daß $N$. affinis stets Nikotin enthielt $(0,84$ bis 3,56\%), wenn sie als Pfropfreis einer Pllanze von $N$. Tabacum mit ungefabr $4 \%$ Nikotingehalt aufsa $B$, oder wenn sie als Unterlage fuir $N$. Tabacum diente. Die Autoren machen auch einen Versach, welcher die Frage entscheiden soll, ob die Fähigkeit von $N$. affinis, Nikotin zu bilden, gesteigert werde, wenn sie mil N. Tabacum verbonden werde. Sie pfropften $N$. Tabacum auf N. affinis Am 8. April schnitten sie das Reis unterhalb der Pfropfotelle ab und ließen die Unterlage $Z$ weige bilden, deren Alkaloidgehalt am 15. Mai $0,33 \%$ betrag. Danach vermuten die Autoren, „daß die Befthigang der Unterlage zur Nikotinbildung durch die Wirkang des nikotinreichen Edelreises gesteigert wir $d^{*}$. Unserer Meinung nach liegt kein Grund zu dieser Vermutung vor. Man könnte, wenn man sich auf die Angaben der Aatoren stittzt, sehr wohl annehmen, daß die 0,3\% Alkaloid eingewandert seien, da ja die Unterlage vor dem Abschneiden des Pfropfreises von letzterem $2,9 \%$ Alkaloid zugeführt erhalten haben könnte. Freilich dârfte man auch annehmen, daß $N$. affinis die $0,3 \%$ Alkaloid selbst gebildet habe.

Wären die Resultate der Versnche von Grafe und Linsbauer einwandfrei, so würden sie beweisen, daß bei zwei nahe verwandten, nikotinbildenden Pflanzen das Nikotin ăaerst leicht durch die Pfropfstelle hindurchwandern kann.

In der anderen der erwahnten Arbeiten teilte ferner H. Lindemath (1906) mit, daß er $1896835 \mathrm{~g}$ Kartoffelknollen, welche durch ein Pfropfreis von Datura Stramonium ernahrt worden waren, von Lewin habe untersuchen lassen, welcher folgendes mitgetellt habe: "Eg würde ihm von großem Interesse sein, za wissen, auf welchem Wege Berr Dr. Klinger das Atropin isoliert hat. Atropin chemisch nachzuweisen sei absolnt unmoglich. Auf einem sehr amstandlichen Wege ließ sich dartun, daß in den Kartoffeln, nach Abtrennung relchlichen Solanins, eine nicht isolierbare Substanz in winzigen Sparen zurtickblieb, die das durch Muskarin zam Stillstand gebrachte Froschherz wieder in Bewegung setzte."

Es leuchtet ein, daB das Erscheinen dieser beiden besprochenen Abhandlungen kein Grund fur ans sein konnte, unseren vorher er- 
wăhnten Plan antzugeben, und wir haben danach zuerst die Untersuchang der Kartoffelknollen in folgender Weise susgefuhrt:

Im Herbst 1906 stand ans also die sehr kraftige Pfropfung von Solanum tuberosum zur Verfügang. Es waren im Mai 1906 aut drei Zweige einer auggetriebenen Kartoffelknolle drei Pfroptreiser von Datura aufgesetzt worden, die ungefahr $80 \mathrm{~cm}$ hoch geworden waren und ungefähr $800 \mathrm{~g}$ bis $7 \mathrm{~cm}$ lange, rundliche Kartoffeln gebildet hatten. Die Bliten der Datura wurden stets entfernt, nur eine gut entwickelte, noch nicht völlig reife Kapsel war bei der Kartoffelernte an den Achsen von Datura vorhanden.

Von den geernteten Kartoffeln diente ein Teil $(410 \mathrm{~g})$ zar Prifung anf mydriatisch wirkende Alkaloide. Die hierzu verwendeten Knollen, welche sich also in ihrem Aeußeren and in ihren Groben darchaus nicht von den normalen Kartoffeln onterschieden, warden zu diesem Zwecke in eine breiartige Masse verwandelt, letztere hierauf mit dem dreifachen Volumen Alkohol von $95 \%$ vermischt and das Gemisch alsdann unter zeitweiligem Umschutteln sechs Tage lang bei einer Temperatur von $20-25^{\circ}$ stehen gelassen. Nach dieser Zeit ist die schwach sauer reagierende Fltussigkeit abkoliert, der Rückstand ausgepreßt und unter den gleichen Bedingungen von neuem mit Alkohol extrahiert worden. Die vereinigten Alkoholausztige wurden hierauf filtriert und durch Destillation im luftverdunnten Ranme von Alkohol befreit.

Der erkaltete Destillationsrückstand (D) wurde abermals filtriert, alsdann im Scheidetrichter mit dem gleichen Volumen Chloroform-Aether (2 Teile Chloroform, 5 Teile Aether) uberschichtet und nach dem $\mathrm{Zu}$ satz von gepulvertem Natriumbikarbonat längere Zeit geschtittelt. Dieses Ausschütteln ist dreimal mit je dem gleichen Volumen Chloroform-Aether wiederholt worden. Die vereinigten Chloroform-Aetherauszüge sind hierauf unter zeitweiligem Aetherzusatz eingedampft worden, bis durch empfindliches rotes Lackmuspapier eine Abgabe von Ammoniak nicht mehr zu konstatieren war. Der Rllckstand wurde hierauf dreimal mit je $5 \mathrm{ccm}$ Wasser, welches schwach mit Salzsäure angesäuert war, ausgeschüttelt und die vereinigten sauren Flüsgigkeiten alsdann mit den allgemeinen Alkaloidreagentien auf Pflanzenbasen gepruft. Diese Priffang fiel jedoch unter Anwendang von je einem Tropfen des sauren Auszuges negativ aus. Erst als dieselbe über Aetzkalk im Vakunm bis auf etwa $2 \mathrm{ccm}$ eingeengt war, konnten schwache Alkaloidreaktionen beobaohtet werden.

Da nach den Erfahrungen, welche von dem einen von uns bei der Isolierung mydriatisch wirkender Alkaloide aus pflanzlichem Material vielfach gemacht wurden, es nicht susgeschlossen war, daß 
die von dem erkalteten Destillationsrickstande (D) abfiltrierten fetthaltigen Massen etwas Alkaloid enthalten konnten, so worden dieselben wiederholt mit Petroleum mit Wasser, dem eine geringe Menge Salzsăare zugefügt war, anegeschuttelt. Diese Auszuge wurden nach dem Verdunsten über Aetzkalk im Vakunm mit den obigen vereinigt.

Zor Identifizierung der anscheinend nur in sehr geringer Menge vorliegenden Alkalolde wurde die Flüssigkeit mit einem Tropfen Goldchloridlösung versetzt and alsdann der frelwilligen Verdunstung uberlassen. Hierbei war die Bildung vereinzelter gelblicher Aggregate von winziger Größe zu beobachten, von Aggregaten, welche eine gewisse Aehnlichkeit mit denen zelgten, die, allerdings in größerem Formate, bei der Verdunstung einer nureinen, in entsprechender Weise aus pflanzlichem Material dargestellten Lösung von Atropin- and Hyoscyamingoldchlorid auftreten. Ein wiederholt anggefuhrter Versuch, diese winzigen Partikelchen nach vorsichtiger Entfernung der kleinen Mengen von Mutterlauge durch Umkrystallisation in die typischen Formen des Atropin bezw. Hyoscyamingoldchlorids tiberzuführen, mißlang, indem an deren Stelle stets nur wenige amorphe, gelbe Flocken resultierten.

Die Chlorotorm-Aetherauszüge, welche bei dem weiteren Ausschutteln des Kartoffelextraktes nach Zusatz von Sodalðsung noch erhalten wurden, lieferten selbst in konzentrierterer Lösung kaum noch Alkaloidreaktionen. Da bei der weiteren Prüfung dieser Auszüge sich anf chemischem Wege noch weniger ein positiver Anhalt tur das Vorhandensein eines mydriatisch wirkenden Alkaloids ergab, als dies bei denen, welche ans dem mit Natriumbikarbonat alkalisierten Kartoffelextrakte resultierten, der Fall war, so wurden beide Lösungen vereinigt, um zur physiologischen Prufung verwendet zu werden. Nach Entfernnng des Goldes aus den gesamten jetzt vorliegenden Lösungen and Ausscheidungen durch Schwefelwasserstoff wurden die Flussigkeiten zu diesem Zwecke im Vaknum Hber Aetzkalk verdanstet und der winzige Ruckstand zur Beseitigung der letzten Salzaäurespuren noch mehrere Tage lang im Vakuumexsikkator über Aetzkalk aufbewahrt. Zur weiteren Reinigung ist der Verdunstungsrdckstand schlieBlich noch mit Alkohol extrabiert in der filtrierten Lösung von neuem im Vakuum verdunstet worden.

Die Berren DDr. A. Lohmann and M. Schenck hatten die Gtite, jenes Produkt im hiesigen physiologischen Institut an dem Auge einer Katze auf seine mydriatische Wirkung zu prüfen. Es konnte jedoch innerhalb einer füntstündigen Beobachtungszeit nicht die geringste Pupillenerweiterung konstatiert werden. 
Da nach den Beobachtangen von Donders and Ruyter') noch durch einen Tropfen einer Atropinlösung 1:130000 Pupillenerweiterung eintritt und auch Hyoscyamin dieselbe Wirkung, nur etwas langsamer, aber um so nachbaltiger, verursacht (Dragendorff l. c.), so ist wohl kaum anzanehmen, dass in den $410 \mathrm{~g}$ der zar Untersuchung benutzten Kartoffeln die Mydriatica in nachweisbarer Menge enthalten waren.

Um weiter einen Anhalt zu gewinnen, wie sich normale Kartoffeln unter den beschriebenen Bedingangen chemisch und physiologisch verhalten, wurde $1 \mathrm{~kg}$ davon in der gleichen Weise einer Prtifang unterzogen. Das Verhalten des erzielten Extraktes war durchans das gleiche wie das der Datura Kartoffelansztige. Die Chloroform-Aetherausschuttelungen lieferten hier eine Flüssigkeit, welche nach Konzentration auf etwa $2 \mathrm{ccm}$ mit den allgemeinen Alkaloidreagentien Reaktionen gab, die unter Berticksichtigung der gröBeren Menge des asgewendeten Untersuchungsmaterials naturgemäß etwas stärker ausfieleu als die frubher beobachteten. Bei der Prüfung mit Goldchlorid traten dieselben Erscheinangen auf, wie dieselben oben beschrieben wurden. Auch hier ließen sich die in geringer Menge ausgeschiedenen gelblichen Aggregate nicht durch Umkrystallisation in eine greifbare Form überführen. Die durch Schwefelwasserstoff wieder von Gold befreiten Lðsungen wurden daher auch in diesem Falle, nach Entfernang der freien Salzsäure und der sonstigen Beimengungen in der im vor. stehenden angegebenen Weise, zur physiologischen Prüfong verwendet. Herr Professor Dr. A. Heffter-Marburg hatte die Güte, letztere auszufthren and als Resultat derselben mitzuteilen, daB sich auch dieses Produkt als ganz wirkangslos auf die Katzenpupille erwiesen hat.

Nach diesen Beobachtungen schien es zunächst nur noch erforderlich za sein, noch den direkten Beweis za erbringen, daß die zum Nachweis des Hyoscyamins angewendete Methode auch den Grad von Zuverlässigkeit und Empfindlichkeit besitzt, welcher für diese $Z$ wecke nötig ist.

$\mathrm{Zu}$ letzterem Zwecke wurde $1 \mathrm{~kg}$ Kartoffeln in der im vorstehenden dargelegten Weise einer erneuten Prufung unterzogen, nachdem dem Kartoffelbrei $2 \mathrm{mg}$ Hyoscysmin zugefögt waren. Die hierbei erzielten Auszage zeigten anch in verdinntem Zustande, d. h. ohne vorherige Konzentration uber Aetzkalk im Vakuum, mit den allgemeinen Alkaloidreagentien deutliche Alkaloidreaktionen.

Zur Identifizierang des vorhandenen Alkaloids mit Hyoscyamin, bez. dessen Umlugernngsprodakt, dem Atropin, warden diese Auszige in zwei gleiche Teile (A und B) geteilt.

1) Dragendorff, Ausmittelung von Giften. 
Teil A wurde in Vakuam tiber Aetzkalk verdunstet, der Ruckstand mit absclatem Alkohol extrahiert and diese Lösung hierant von nenem im Vakuam verdampft. Mit dem Verdanstangsrückstand warde alsdann die Vitali'sche Reaktion anggefuhrt. Dieselbe trat in einwandfreier Weise ein.

Tell B wurde mit einem Tropfen Goldchloridlösung versetzt und alsdann der freiwilligen Verdunstung therlassen. Es gelangten hierbet kleine gelbe Aggregate zar Ausscheidung, die in dem Aeußern durchaus an die erinnerten, welche bel der Verdunstung einer unreinen, in entsprechender Weise ans pflanzlichem Material dargestellten Losang von Atropin-bezw. Hyoscyamingoldchlorid auftreten. Nach Entfernung der Mutterlauge traten bei vorsichtigem Umkrystallisieren aus schwach salzsăurehaltigem Wasser diese éigenartigen Formen von nenem auf. Zor Ermittelung des Schmelzpunktes war jedoch die Menge dieser Ausscheidungen $\mathrm{zu}$ gering.

Zur weiteren Kennzeichnong wurden daher diese Aggregate in Wasser gelöst, diese Lösung im Verein mit der Mutterlauge durch Schwefelwasserstoff von Gold befreit und die filtrierte Flüssigkeit von neuem über Aetzkalk in Vakuum verdunstet. Nach weiterer Reinigung durch Extraktion mit absolutem Alkohol and erneutes Verdunsten resultierte schließlich ein Rückstand, der zur physiologischen Prüfung Verwendung fand.

Die Herren DDr. A. Lohmann und M. Sckenck hatten die Güte, auch dieses Produkt im hiesigen physiologischen Institut an dem Auge einer Katze auf seine mydriatische Wirkung zu prifen. Nach Verlauf von 20 Minaten konte hierbei eine deutliche Pupillenerweiterung konstatiert werden.

Erwăgt man, daß der mit $2 \mathrm{mg}$ Hyoscyamin versetzte $1 \mathrm{~kg}$ betragende Kartoffelbrei nur einmal mit der dreifachen Menge Alkohol extrahiert und abgepreßt war, und berïcksichtigt man die bei dieser Operation nnvermeidlichen Verluste und teilweisen Zersetzungen des angewendeten Alkaloids, so erhellt, daß nach dem angewendeten Untersuchungsverfahren sich in $500 \mathrm{~g}$ Kartoffeln noch weniger als I mg Hyoscyamin, sowohl chemisch, als anch physiologisch nach. weisen läßt.

Wenn die früher zur Untersuchung verwendeten Datura-Kartoffeln daher überhaupt Hyoscyamin enthielten, so durfte nach diesen Erfahrungen die Menge jenes Alkaloids für die zur Prüfung benutzten $410 \mathrm{~g}$ weit weniger als $1 \mathrm{mg}$ betragen haben.

Einstweilen ist die Frage, ob Hyoscyamin ans dem Pfropfreis in die Unterlage wandert, im negativen Sinne za beantworten. 
Da jedoch die Angaben von Klinger und anch die von Lewin in gewisser Weise ${ }^{1}$ ) unserer Erfahrung entgegenstehen, so wollen wir unsere Untersuchung, wenn es angeht unter Anwendung einer noch größeren Menge von Kartoffeln, nochmals wiederholen, obschon wir uns überzengt haben, daß sich mit unserer Methode eine außerst kleine Hyoscyaminmenge in Kartoffeln nachweisen last. Ferner werden wir noch folgende Fragen zu entscheiden versuchen:

1. Da wir wissen, daß aus Blattstielen von Datura das Byoscyamin verschwindet, werden wir fragen, ob vielleicht aus absterbenden Pfropfreisern von Datura Hyoscyamin in die Unterlage wandert.

2. Wir werden ferner zu entscheiden versuchen, ob Hyoscyamin ans entblätterten Pfropfreisern von Datura auswandert.

3. Es soll untersucht werden, ob Nikotin aus Pfropfreisern von Nicotiana Tabacum und rustica in die als Unterlage benatzte Kartoffelpflanze einwandert.

4. Wir wollen eventuell Versuche darúber anstellen, ob die Alkaloide der Pfropfreiser in der Unterlage verăndert werden.

5. In allen Versuchen soll die Pfropfstelle mikrochemisch auf die Lagerung der Alkaloide geprlfft werden.

\section{Literatar.}

Feldhaus, Quantitative Untersucbung der Verteilung des Alkaloides in den Organen von Datura Stramonium, Dissertation, Marburg 1903.

Kircher, Ueber das mydriatisch wirkende Alkaloid der Datura metel, Datura quercifolia, Datura arborea, Dissertation, Marburg 1905.

Siim Jensen, Beitrăge zur botanischen and pharmakognostischen Kenntnis ron Hyoscyamus niger L., Bibliotheca botanica Heft 51, 1901; Arbeit aus dem botanischen Institute der Universitat Marburg.

H. Lindemuth, Ueber angebliches Forhandensein ron Atropin in Kartoffetknollen infolge von Transplantation und uber die Grenzen der Verwachsung Dach dem Verwandtschaftsgrade; Berichte der Deutschen Botanischen Gesellschaft 1906, S. 428

E. Strasbarger, Ueber Verwachsung and deren Folgen; Berichte der Deutschen Botanischen Gesellschaft 1885, S. XXXIV,

E Strasburger, $\mathrm{Zu}$ dem Atropinnachweis in den Kartoffelknollen; Berichte der Dentschen Botanischen Gesellscbaft 1906, S. 699.

V. Grafe and K. Linsbauer, Ueber die wechselseitige Beeinflussnng von Nicotiana Tabacum und $N$. affinis bei der Pfropfung; Berichte der Deutschen Botanischen Gesellschaft 1906, S. 366.

1) Es ist dabei zu beachten, daB in der Literatur Angaben vorliegen, daB der Muskarinstillstand auch durch andere Stoffe, wie Guanidin, Camphor, Veratrin, Digitalin usw. aufgehoben werden konne, sodaB es nicht ganz sicher ist, dab der Stillstand wirklich durch Hyoscyamin herbeigeführt wurde. 